\title{
Espacios de decisión en la descentralización de salud del Paraguay
}

\author{
Edgar Giménez Caballero, José Carlos Rodríguez, Néstor Peralta
}

Investigación para el Desarrollo. Paraguay

Cómo referenciar este artículo/

How to reference this article:

\begin{abstract}
Giménez E, Rodríguez JC, Peralta N. Espacios de decisión en la descentralización de salud del Paraguay. Mem. Inst. Investig. Cienc. Salud. $2016 ; 14(3): 44-51$
\end{abstract}

\section{R E S U M E N}

Aunque está establecida en las leyes, la descentralización se desarrolla con lentitud en Paraguay. Este artículo la evaluó en la salud pública, desde el marco conceptual de los Espacios de Decisión donde El Principal tomador de decisiones es el Ministerio de Salud, mientras que sus Agentes son los establecimientos y Consejos de Salud. Se entrevistaron a 511 personas del sistema público de salud. Las funciones evaluadas fueron: administración de recursos financieros y humanos, gestión de servicios mediante reglas de dirección y organización, administración de reglas de acceso para mejorar la accesibilidad, y participación social en torno a los servicios de salud. Se incluyeron en el estudio a las capitales departamentales de la Región Oriental y al Departamento de Presidente Hayes de la Región Occidental. La descentralización de la salud pública paraguaya se encontró que estaba entre mínima e incipiente. La mayor descentralización estuvo en la función de organización, sugiriendo una opción local a favor del proceso, seguida por la de dirección, el acceso, los recursos humanos y la participación. La menor descentralización en Finanzas puede relacionarse con la carencia crónica de recursos fiscales del Estado.

Palabras claves: Descentralización, Salud Pública, Consejos de Salud, Paraguay, Espacios de Decisión.

\section{Decision spaces on health decentralization in Paraguay}

\section{A B S T R A C T}

Although decentralization it is established by the law, it develops slowly in Paraguay. This article evaluates public health decentralization from the conceptual framework of Decision Spaces where the major decision maker is the Ministry of Health while its Agents are Health establishments and Councils. Five hundred eleven people from the public health system were interviewed. The decentralized functions studied were: management of financial and human resources, service management by rules of organization and direction, access rules management to improve accessibility and social participation in relation to health services. In this study, the departmental capitals of the Eastern Region and the capital of the department of President Hayes in the Western Region were included. The decentralization of the Paraguayan public health was between Minimum to Incipient. The greater decentralization was in the organization function, suggesting a local option in favor of the process, followed by direction, access, human resources and participation. The lower decentralization in Finances can be related to the chronic lack of fiscal resources of the state.

Keywords: Decentralization, Public Health, Decentralization, Public Health, Health Councils, Paraguay, Decision Spaces. 


\section{INTRODUCCIÓN}

La descentralización fue una de las reformas del Estado que tuvo lugar en varios países de América Latina desde los años '80, bajo distintas modalidades y especificidades, dependientes de cada contexto.

En Paraguay, el proceso adquirió una sólida base jurídica con la Constitución Nacional de 1992 que declaró de manera expresa, en el Artículo 1, que el país es una República Unitaria y Descentralizada (1). La descentralización política se estableció a través de las Gobernaciones y las Municipalidades. A partir de la vigencia de esta Constitución durante la década de los '90, se promulgaron leyes para la descentralización política del Estado Paraguayo y para la descentralización sectorial de la salud.

La Ley de la "Carta orgánica del Gobierno Departamental" (2) estableció que servicios públicos de carácter nacional podrían ser trasferidos a la administración departamental a iniciativa de las propias gobernaciones. La ley "Orgánica Municipal" (3) estableció funciones específicas a las comunas en materia de salud, higiene y salubridad.

En el campo de la salud, mediante la Ley del "Sistema Nacional de Salud" (4), se creó la figura de los Consejos Locales y Regionales de salud; un Consejo Nacional y tres Direcciones Ejecutivas para conducir el Plan Nacional de Salud.

En este contexto, en la descentralización del sector salud, se desarrollaron tres procesos: a) La generación de espacios de participación social a nivel departamental y municipal mediante los Consejos de Salud; b) Espacios departamentales y municipales para la gestión sanitaria mediante Secretarías de Salud de las Gobernaciones y Direcciones correspondientes de las Municipalidades; y, c) Desconcentración administrativa del Ministerio de salud (5-7).

Este estudio analiza el grado de descentralización del Ministerio de Salud Pública y Bienestar Social, el que se ha considerado como Principal, en tanto que los Establecimientos de Salud dependientes y los Consejos de Salud relacionados se consideraron sus Agentes de descentralización (8).

Para el análisis se adoptó el marco conceptual de "espacios de decisión". La descentralización se concibe como una variedad de reformas caracterizadas por la transferencia de autoridad fiscal, administrativa y/o política del Ministerio de Salud central hacia instituciones alternativas. Estas instituciones receptoras pueden ser direcciones locales o regionales del mismo ministerio, gobiernos municipales, agencias de servicio público autónomas u organizaciones del sector privado. Eso permite a las entidades descentralizadas tomar decisiones en un rango posible de opciones en distintas funciones de servicios de salud: a) administración de recursos financieros, humanos y bienes; b) gestión de servicios mediante reglas de dirección y organización c) administración de reglas de acceso para mejorar la accesibilidad de los servicios; y d) la participación social en torno a los servicios de salud. El poder de decisión en las opciones posibles se considera espacios de decisión en la descentralización de salud. (9).

\section{MATERIALES Y MÉTODOS}

La población en estudio corresponde a personas relacionadas a la toma de decisiones en procesos de las funciones de espacios de decisión estudiadas en la descentralización o desconcentración del Ministerio de Salud Pública y Bienestar Social identificadas con un mapeo de actores. Los criterios de inclusión fueron los siguientes: Directores y profesionales de salud con jefatura o responsabilidad en la gestión de Direcciones Regionales, Hospitales Regionales y Hospitales Distritales; Directores y profesionales de salud de Centros de Salud y Unidades de Salud de la Familia; e c) Integrantes de Consejos de Salud relacionados.

Las localidades seleccionadas para el estudio fueron: a) Capitales de los Departamentos de la Región Oriental y el Departamento de Presidente Hayes de la Región Occidental; b) Distritos donde se asientan hospitales dependientes del Ministerio de Salud Pública y Bienestar Social; c) Distritos inmediatamente aledaños a los hospitales donde asientan Centros de Salud y Unidades de Salud de la Familia.

Incluyó el estudio a informantes claves de 15 Direcciones Regionales, 15 Hospitales Regionales, 39 hospitales distritales, 10 Centros de Salud, 8 Unidades de salud familiar y a 54 Consejos de Salud. En cada establecimiento dependiente del Ministerio de Salud Pública y Bienestar Social se incluyó al Director General, a otros 2 Directores de nivel y a un profesional con responsabilidad de gestión. En los Consejos de Salud se incluyó a 3 integrantes. La muestra por conveniencia tiene 511 informantes claves que respondieron cuestionario sobre el poder que tienen los Agentes locales, en relación al Principal, para 
decidir sobre funciones de gestión de servicios de salud. El levantamiento de datos se realizó entre setiembre y diciembre de 2015. Las variables en cada función fueron las siguientes:

a) En la administración de recursos financieros: el poder de decisión para elaborar, gastar, priorizar e incorporar inversiones en el presupuesto, y en la administración de recursos humanos el poder de decisión para incorporar, distribuir horarios, trasladar, sancionar, despedir o incentivar a los funcionarios; b) En la gestión de servicios mediante la dirección se consideró: el poder para designar directores, planificar, establecer metas, controlarlas, rendir cuentas y contrarrestar las influencias partidarias en sus decisiones mientras que en la función de organización de servicios se consideró el poder de decisión para mejorar la calidad, asignar tareas, proveer servicios prioritarios para su comunidad y utilizar información para tomar decisiones; c) En la función reglas de acceso: el poder de decisión para identificar a la población más vulnerable, mejorar el acceso de la población indígena, pobres, personas con discapacidad, de la tercera edad, mujeres y niños; finalmente, d) En la función de participación social capacidad para planificar, mejorar la calidad, en actividades de promoción de la salud y de prevención, en situaciones de emergencia y en la rendición de cuentas.

Para estandarizar las respuestas se utilizó una escala de Likert de 1 a 4 . El mínimo poder de decisión fue 1 y el máximo poder de decisión 4. Los resultados fueron procesados para describir la distribución de frecuencias y las proporciones. Se calculó el total de puntos en cada respuesta para cada componente de la función, en relación a los puntos máximos posibles*. Esta proporción indica el desempeño del espacio de decisión (DED) para cada componente. Se asume que cada componente posee el mismo peso relativo que los demás. Los espacios de decisión fueron categorizados como mínimos, incipientes, moderados y máximos. Para traducir los puntajes de Likert a estas categorías se tomó una amplitud de 18,75 , con valor mínimo 25 . El valor máximo es 100 , el rango fue de 75 con 4 intervalos que se corresponden con la escala utilizada en el cuestionario.

*100 $\times[(n \times 1)+(n \times 2)+(n \times 3)+(n \times 4)] /(N \times 4)]$ En donde: $n$ es la nota que cada encuestado da a cada característica. $\mathrm{N}$ es la suma de las características evaluadas. Los números 1, 2, 3, 4 son las notas posibles asignables.

\section{RESULTADOS}

En el estudio se incluyó a los 511 cuestionarios válidos, 74\% directores de establecimientos o de ese nivel; $11 \%$ fueron profesionales de salud y $15 \%$ integrantes de Consejos de Salud. Los resultados son presentados en su distribución de frecuencias y el Desempeño del Espacio de Decisión DED para cada componente de las funciones. En la tabla 1 se presentan los resultados sobre el poder de decisión para el manejo de recursos financieros y de recursos humanos. 
Tabla 1. Descentralización de salud del Paraguay. Poder de decisión de los Agentes para el manejo de recursos Financieros y Humanos. Escala de Likert de 1 (mínimo) a 4 (máximo) Desempeño del espacio de decisión (DED): 100*total de puntos, 4 total de puntos posibles.

\begin{tabular}{|c|c|c|c|c|c|c|c|c|c|c|}
\hline \multirow{2}{*}{ Componentes/Función } & \multirow{2}{*}{$\mathbf{n}$} & \multicolumn{2}{|c|}{1} & \multicolumn{2}{|c|}{2} & \multicolumn{2}{|c|}{3} & \multicolumn{2}{|c|}{4} & \multirow{2}{*}{ DED } \\
\hline & & $n$ & $\%$ & $n$ & $\%$ & $\mathbf{n}$ & $\%$ & $\mathrm{n}$ & $\%$ & \\
\hline Formular el Presupuesto & 510 & 260 & 50,9 & 136 & 26,6 & 98 & 19,2 & 16 & 3,1 & 43,6 \\
\hline Gastar el Presupuesto & 511 & 265 & 51,9 & 158 & 30,9 & 76 & 14,9 & 12 & 2,3 & 41,9 \\
\hline Establecer prioridades & 511 & 218 & 42,7 & 162 & 31,7 & 115 & 22,5 & 16 & 3,1 & 46,5 \\
\hline Incluir plan de inversiones & 511 & 220 & 43,1 & 153 & 29,9 & 121 & 23,7 & 17 & 3,3 & 46,8 \\
\hline Función financiera & 2043 & 964 & 47,2 & 611 & 29,9 & 413 & 20,2 & 65 & 3,2 & 45,1 \\
\hline Incorporar & 510 & 235 & 46 & 176 & 34,4 & 79 & 15,5 & 20 & 3,9 & 44,3 \\
\hline Asignar funciones & 510 & 113 & 22,1 & 130 & 25,4 & 175 & 34,2 & 91 & 17,8 & 61,9 \\
\hline Establecer carga horaria & 511 & 116 & 22,7 & 120 & 23,5 & 168 & 32,9 & 107 & 20,9 & 63 \\
\hline Promover e incentivar & 511 & 131 & 25,6 & 171 & 33,5 & 171 & 33,5 & 38 & 7,4 & 55,7 \\
\hline Sancionar & 510 & 176 & 34,4 & 151 & 29,5 & 148 & 29 & 35 & 6,8 & 52,1 \\
\hline Movilizar o trasladar & 510 & 179 & 35 & 156 & 30,5 & 140 & 27,4 & 35 & 6,8 & 51,5 \\
\hline Desvincular & 511 & 220 & 43,1 & 189 & 37 & 87 & 17 & 15 & 2,9 & 45 \\
\hline Función recursos humanos & 3573 & 1170 & 32,7 & 1093 & 30,6 & 968 & 27,1 & 341 & 9,5 & 53,3 \\
\hline
\end{tabular}

Fuente: Proyecto: Espacios de decisión en la descentralización de salud del Paraguay. Investigación para el Desarrollo. Año 2016. Financiado por PROCIENCIA - CONACYT

El espacio de decisión para administrar los recursos financieros a nivel local fue valorado entre mínimo e incipiente. El poder de decidir sobre la elaboración del presupuesto y para gastar el presupuesto tiene el mínimo espacio de decisión. El poder para establecer prioridades en la asignación de recursos y de incorporar al presupuesto un plan de inversiones locales fue valorado con incipientes espacios de decisión.

En general, el poder para decidir sobre distintos aspectos de la administración de recursos humanos fue valorado con incipientes espacios de decisión; en tanto que el poder para establecer la distribución de la carga horaria en el establecimiento fue valorado como un espacio de decisión moderado.

En general, el poder para decidir sobre distintos aspectos de la administración de recursos humanos --como incorporar nuevos profesionales y funcionarios, asignar funciones al personal, incentivarlos y promoverlos, sancionarlos, trasladarlos o desvincularlos-fue valorado con incipientes espacios de decisión; en tanto que el poder para establecer la distribución de la carga horaria en el establecimiento fue valorado como un espacio de decisión moderado.

En la tabla 2 se presentan los resultados sobre el poder de decisión para la gestión, donde los espacios de decisión se amplían, sobre todo, en aquellos componentes más funcionales como son: planificar, establecer metas de gestión, controlar el cumplimiento de estas metas, asignar funciones para organizar mejor los servicios y utilizar la información generada para tomar decisiones. Estos componentes de las funciones de dirección y organización de servicios fueron valorados con poder moderado. En cambio, los componentes políticos y administrativos de estas funciones fueron valorados como espacios de decisión incipiente. 
Tabla 2. Descentralización de salud del Paraguay. Poder de decisión de los Agentes para la gestión de servicios: Dirección y Organización. Escala de Likert de 1 (mínimo) a 4 (máximo). Desempeño del espacio de decisión (DED): 100*total de puntos, 4 total de puntos posibles.

\begin{tabular}{|c|c|c|c|c|c|c|c|c|c|c|}
\hline \multirow{2}{*}{ Componentes/Función } & \multirow{2}{*}{$n$} & \multicolumn{2}{|c|}{1} & \multicolumn{2}{|c|}{2} & \multicolumn{2}{|c|}{3} & \multicolumn{2}{|r|}{4} & \multirow{2}{*}{ DED } \\
\hline & & $\mathbf{n}$ & $\%$ & $\mathbf{n}$ & $\%$ & $\mathbf{n}$ & $\%$ & $\mathbf{n}$ & $\%$ & \\
\hline Elaborar planes & 510 & 89 & 17,4 & 124 & 24,3 & 221 & 43,2 & 76 & 14,9 & 63,9 \\
\hline Fijar metas de gestión & 511 & 76 & 14,9 & 127 & 24,9 & 228 & 44,6 & 80 & 15,7 & 65,3 \\
\hline Controlar metas & 511 & 71 & 13,9 & 138 & 27 & 246 & 48,1 & 56 & 11 & 64 \\
\hline Rendición de cuentas & 511 & 159 & 31,1 & 188 & 36,8 & 129 & 25,2 & 35 & 6,8 & 52 \\
\hline Designar directores & 510 & 161 & 31,5 & 155 & 30,3 & 141 & 27,6 & 53 & 10,4 & 54,2 \\
\hline Contrarrestar influencia & 511 & 186 & 36,4 & 207 & 40,5 & 90 & 17,6 & 28 & 5,5 & 48 \\
\hline Función de dirección & 3064 & 742 & 24,2 & 939 & 30,6 & 1055 & 34,4 & 328 & 10,7 & 57,9 \\
\hline Asignar funciones & 511 & 81 & 15,9 & 107 & 20,9 & 224 & 43,8 & 99 & 19,4 & 66,7 \\
\hline Nuevos servicios & 510 & 122 & 23,9 & 151 & 29,5 & 204 & 39,9 & 33 & 6,5 & 57,3 \\
\hline Programas prioritarios & 511 & 98 & 19,2 & 129 & 25,2 & 238 & 46,6 & 46 & 9 & 61,4 \\
\hline Servicios prioritarios & 510 & 88 & 17,2 & 151 & 29,5 & 228 & 44,6 & 43 & 8,4 & 61,1 \\
\hline Generar y utilizar información & 511 & 64 & 12,5 & 96 & 18,8 & 250 & 48,9 & 101 & 19,8 & 69 \\
\hline Mejora de calidad & 511 & 95 & 18,6 & 109 & 21,3 & 266 & 52,1 & 41 & 8 & 62,4 \\
\hline Función de organización & 3064 & 548 & 17,9 & 743 & 24,2 & 1410 & 46 & 363 & 11,8 & 63 \\
\hline
\end{tabular}

Fuente: Proyecto: Espacios de decisión en la descentralización de salud del Paraguay.

Investigación para el Desarrollo. Año 2016. Financiado por PROCIENCIA - CONACYT

Incipiente es también la capacidad de decidir para implementar nuevos programas y servicios o aquellos que son prioritarios para la comunidad local o bien para diseñar e implementar planes de mejora de la calidad.

En la Tabla 3 se presentan los resultados sobre el poder de decisión para establecer las reglas de acceso para la población más vulnerable local. A nivel local el poder para identificar a la población más vulnerable es moderado. En cambio, este poder se reduce a incipiente cuando tienen que implementar acciones concretas para facilitar el acceso de los mismos.

Tabla 3. Descentralización de salud del Paraguay. Poder de decisión para mejorar el acceso a grupos vulnerables. Escala de Likert de 1 (mínimo) a 4 (máximo). Desempeño del espacio de decisión (DED): $100 *$ total de puntos, 4 total de puntos posibles.

\begin{tabular}{|c|c|c|c|c|c|c|c|c|c|c|}
\hline \multirow{2}{*}{ Componentes/Función } & \multirow{2}{*}{$\mathbf{n}$} & \multicolumn{2}{|c|}{1} & \multicolumn{2}{|c|}{2} & \multicolumn{2}{|c|}{3} & \multicolumn{2}{|c|}{4} & \multirow{2}{*}{ DED } \\
\hline & & $\mathbf{n}$ & $\%$ & $\mathbf{n}$ & $\%$ & $n$ & $\%$ & $n$ & $\%$ & \\
\hline Identificar vulnerables & 511 & 80 & 16 & 104 & 20 & 243 & 48 & 84 & 16 & 66 \\
\hline Acceso a mujeres & 511 & 102 & 20 & 148 & 29 & 219 & 43 & 42 & 8,2 & 60 \\
\hline Acceso a niños & 511 & 109 & 21 & 145 & 28 & 211 & 41 & 45 & 8,8 & 59 \\
\hline Acceso a 3ra edad & 511 & 124 & 24 & 154 & 30 & 192 & 38 & 41 & 8 & 57 \\
\hline Acceso p/ discapacidad & 511 & 140 & 27 & 162 & 32 & 173 & 34 & 36 & 7 & 55 \\
\hline Acceso para indígenas & 511 & 193 & 38 & 140 & 27 & 145 & 28 & 33 & 6,5 & 51 \\
\hline Acceso para pobres & 511 & 143 & 28 & 140 & 27 & 196 & 38 & 32 & 6,3 & 56 \\
\hline Función Reglas de Acceso & 3577 & 891 & 24,9 & 993 & 27,8 & 1379 & 38,6 & 313 & 8,8 & 57,8 \\
\hline
\end{tabular}

Fuente: Proyecto: Espacios de decisión en la descentralización de salud del Paraguay.

Investigación para el Desarrollo. Año 2016. Financiado por PROCIENCIA - CONACYT

En la Tabla 4 se presentan los resultados sobre el poder de decisión que tiene la participación social, en los Consejos de Salud, sobre distintos aspectos del funcionamiento de servicios de salud a nivel local.

La participación social es percibida como incipiente en la planificación participativa comunitaria y en definir prioridades locales desde un enfoque comunitario, para incidir en una mejor organización y calidad de servicios y en la rendición de cuentas. Igual valoración tiene la movilización comunitaria para participar en situaciones de emergencia, para realizar actividades de prevención de enfermedades y promoción de la salud. 
Tabla 4. Descentralización de salud del Paraguay. Poder de decisión en la función de participación social. Escala de Likert de 1 (mínimo) a 4 (máximo). Desempeño del espacio de decisión (DED): 100*total de puntos/total de puntos posibles.

\begin{tabular}{llllllllllll}
\hline \multirow{2}{*}{ Componentes/Función } & \multirow{2}{*}{$\mathrm{n}$} & \multicolumn{3}{c}{1} & \multicolumn{2}{c}{2} & \multicolumn{3}{c}{3} & \multicolumn{2}{c}{4} \\
\cline { 3 - 11 } & & $\mathrm{n}$ & $\%$ & $\mathrm{n}$ & $\%$ & $\mathrm{n}$ & $\%$ & $\mathrm{n}$ & $\%$ & \\
\hline Definición de prioridades & 511 & 124 & 24,3 & 262 & 51,3 & 110 & 21,5 & 15 & 2,9 & 50,8 \\
Planificación & 510 & 131 & 25,6 & 243 & 47,6 & 127 & 24,9 & 9 & 1,8 & 50,7 \\
Rendición de cuentas & 510 & 145 & 28,4 & 233 & 45,6 & 117 & 22,9 & 15 & 2,9 & 50,1 \\
Situaciones de emergencia & 510 & 143 & 28,0 & 203 & 39,7 & 145 & 28,4 & 19 & 3,7 & 52,0 \\
Promoción y prevención & 510 & 88 & 17,2 & 239 & 46,8 & 161 & 31,5 & 21 & 4,1 & 55,5 \\
Organización & 510 & 97 & 19,0 & 225 & 44,0 & 160 & 31,3 & 28 & 5,5 & 55,8 \\
Mejora de la calidad & 510 & 98 & 19,2 & 241 & 47,2 & 149 & 29,2 & 20 & 3,9 & 54,3 \\
\hline
\end{tabular}

Fuente: Proyecto: Espacios de decisión en la descentralización de salud del Paraguay. Investigación para el Desarrollo. Año 2016. Financiado por PROCIENCIA - CONACYT

En el Figura 1 se describe el mapa de espacios de decisión por funciones de servicios en la descentralización de servicios de salud.

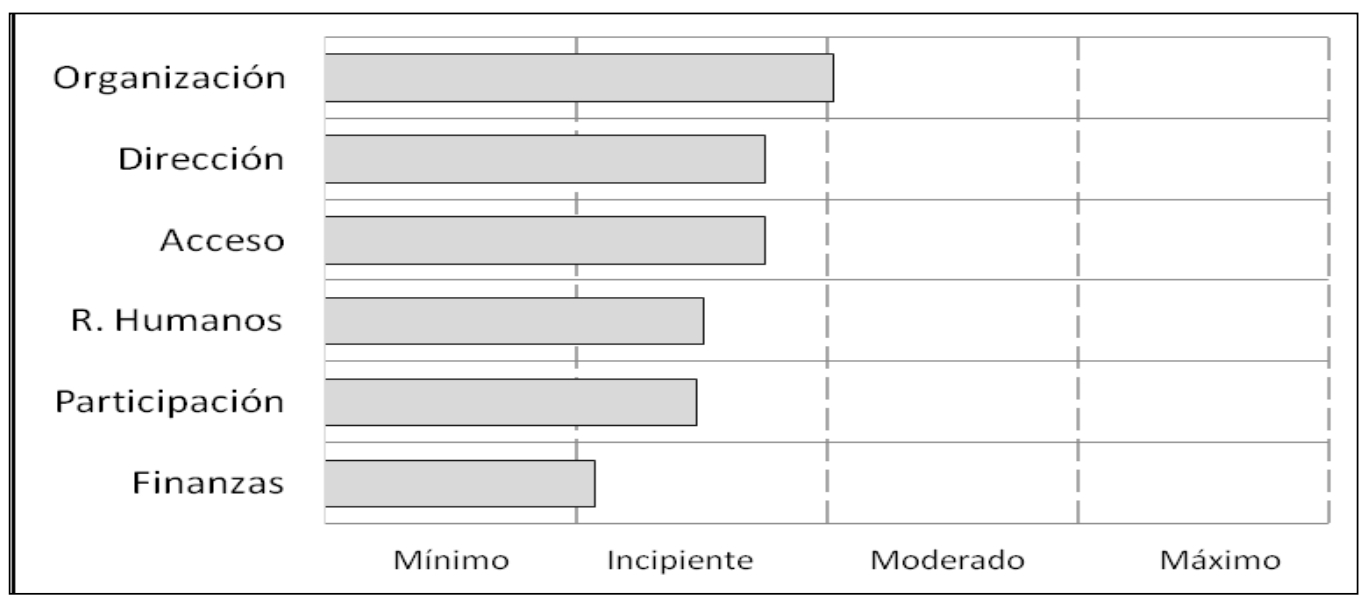

Fuente: Proyecto: Espacios de decisión en la descentralización de salud del Paraguay. Investigación para el Desarrollo. Año 2016. Financiado por PROCIENCIA - CONACYT

Figura 1. Mapa de espacios de decisión en la descentralización de servicios de salud del Paraguay por funciones de servicios.

El espacio de decisión más reducido se da en el poder de decisión y control sobre los recursos financieros y el presupuesto para producir los servicios de salud a nivel local. La función con mayor descentralización es la de organización de servicios salud, que, según esta categorización alcanza un desempeño límite para ser considerada con un poder moderado para decidir sobre sus componentes. En general, las principales funciones como manejo de recursos financieros y humanos, dirigir y establecer reglas de acceso fueron valoradas en un rango incipiente de descentralización, considerando el poder de decisión o el espacio de decisión en cada una de ellas. Lo mismo sucede con la participación social cuya valoración general también es incipiente.

\section{DISCUSIÓN}

Las reformas más notables en el Sistema de Salud del Paraguay han sido: a) en el modelo de gestión: el marco normativo para la descentralización de servicios; b) en el modelo de atención: la estrategia de Atención Primaria de la Salud mediante la instalación de Unidades de Salud de la Familia; y c) en el modelo de financiamiento: la eliminación de aranceles como cuota de recuperación de financiamiento del sistema público (10). El proceso de descentralización supone transferencia de atribuciones medios y capacidades, desde un Principal hacia sus Agentes. No conforma un proceso simple, la tradición del centralismo había sido predominante y no lo favorece. Que el aspecto financiero sea el menos centralizado (mínimo a incipiente) puede estar influenciado por los escases de 
medios locales: presión tributaria del país baja (13\%) y altamente concentrada en el gobierno central $(91,3 \%)(11)$. En el otro extremo, el mayor avance de la descentralización en la Organización (incipiente a moderado) y Dirección (incipiente) puede ser entendido como un reclamo local de búsqueda de auto gestión y auto organización desde abajo, donde ello es posible. Pero estos procesos no están ajenos a la influencia de la política partidaria local, lo que puede favorecer el clientelismo. La menor descentralización en la administración de los Recursos Humanos (incipiente) depende por una parte del manejo central del Presupuesto, pero también implica una forma muy efectiva de ejercer el poder del nivel central, ya que los servicios de salud no se acumulan, sino que se producen y consumen al mismo tiempo que interactúan los profesionales con la población. La Participación Social a nivel municipal es más fácil, en cuanto que los ciudadanos están más cerca de sus autoridades y está formalizada en los consejos de salud. La contrapartida estatal se limita a la trasferencia de fondos mínimos a los consejos para apoyar la gestión de servicios locales, pero la decisión de qué hacer con ellos depende de las autoridades sanitarias locales quienes a su vez dependen de las directrices y normativas del nivel central. El Acceso de los grupos vulnerables, depende en la práctica de las Unidades de Salud Familiar (USF), ya que a nivel local no tienen en sus manos medios para aumentar la inclusión de los pobres, los indígenas, las personas con discapacidad, las personas de la tercera edad, de las mujeres ni de los niños. Esta priorización o focalización depende del Principal. En conclusión, a pesar de contar con un marco legal favorable para la descentralización, una estructura administrativa montada en todos los departamentos y la conformación de consejos de salud en prácticamente todos los distritos, el proceso de descentralización en salud ha sido lento, persistiendo el modelo centralista y altamente burocrático.

Paraguay es un país menos descentralizado que sus vecinos de la región latinoamericana. Eso tiene que ver con varias cuestiones. El pasado autoritario tendía a concentrar la autoridad; La concentración espacial de la población favorecía la concentración de la autoridad. Y, finalmente, el tamaño de país, que posibilitaba la concentración.

El poblamiento cambió los datos. La capital tiene el $8 \%$ de la población y no creció hace 20 años. La marcha hacia el este (y al sur), la del eje norte de la región oriental y, más recientemente, el mayor poblamiento del Chaco han desplazado las densidades poblacionales. Una descentralización óptima no podrá equipararse a la conveniente y real en países mayores en población y superficie, como Brasil, México, Argentina o Colombia. Pero, Paraguay tiene una superficie mucho mayor a los países pequeños y centralizados, como Panamá, el Salvador, Guatemala o Uruguay y eso particulariza la situación. La revolución democrática y los reclamos sociales levantados están cambiando las situaciones. La población y en particular la más cercana al sistema de salud, reclama descentralización, desde las formas más débiles de la 'desconcentración' hasta las más fuertes de la 'devolución' (de poderes). La inequidad social y la territorial se ven juntas, y abiertamente contrarrestadas con la descentralización.

El Sistema de Salud está compuesto de tres paradigmas. El hospitalario, el de la descentralización y el preventivo. Y tiene tres sistemas: el privado, el de los seguros para los asalariados y el de la salud pública. Una optimación de los recursos de la salud requiere la articulación de estos paradigmas y sistemas. Eso supone una coordinación en red que permita hacer circular al paciente que lo necesite entre las instancias más cercanas y de menor complejidad, a las instancias menos cercanas con mayor complejidad. Esto implica una profundización de la descentralización y el desarrollo de otra cultura de la salud: La creación de una mayor "ciudadanía" y cultura sanitaria, con mayor participación, que, a través de los diferentes sistemas, el privado, el de seguros y el público no contributivo, pueda materializar a la salud como derecho, comenzando por universalizar la atención de menor complejidad. La descentralización no es un fin sino un medio para alcanzar objetivos sanitarios, entre ellos los que son intrínsecos a todo sistema de salud: mejorar la salud de la población, contribuir a la equidad y satisfacer las expectativas de la población (12).

Los determinantes sociales influyen en el proceso de búsqueda y atención adecuada. Los esfuerzos deberían orientarse a que la oferta del sistema de salud logre satisfacer las necesidades de salud de la población, en cada comunidad, en cada municipio y departamento del país. Lograr la cobertura universal para prevenir y tratar enfermedades de manera adecuada, oportuna y con calidad, así como proteger contra los riesgos financieros que esto implica es uno de los desafíos más importantes del sistema de salud (13). 
Propuestas a ser consideradas son: Profundizar la desconcentración del Ministerio Salud hacia las Regiones Sanitarias y fortalecer los mecanismos de articulación intersectorial a nivel departamental y municipal; Implementar de un programa de salud pública para dar cobertura a necesidades poco atendidas en el modelo actual y que son prioritarias en la nueva agenda de salud, con énfasis en promoción y prevención de enfermedades; Y, finalmente, iniciar el debate sobre la transferencia de recursos y competencias de algunos servicios personales hacia las gobernaciones y las municipalidades con énfasis en el primer nivel de atención, fomentando la participación social (14).

\section{REFERENCIAS BIBLIOGRÁFICAS}

1. Paraguay. Constitución Nacional 1992. Disponible en: http://jme.gov.py/transito/leyes/cn1992.ht $\mathrm{ml}$

2. Paraguay. Ley No 426/92 Carta orgánica del Gobierno Departa-mental.1992. Recuperada en junio 2016, Disponible en: http://pdba.georgetown.edu/Decen/Paragua y/py_ley426.pdf

3. Paraguay. Ley 3966/10 Orgánica Municipal. 2010 Recuperado en junio 2016, Disponible en: http://www.matusdubarry.com/v2/index.php/leyes/187-ley396610-organica-municipal.

4. Paraguay. Ley 1032/96 del Sistema Nacional de Salud. 1996 Recupera-do en junio 2016, Disponible en http:// www.mspbs.gov.py/ dgds $/ ? p=24$

5. Giménez E. Notas sobre la descentralización en el sector salud del Paraguay. Instituto Desarrollo Asunción; 2012. Disponible en: http://www.desarrollo.org.py/publicacion.ph $\mathrm{p}$ ?id $=85$

6. Mancuello JN, Cabral MS. Sistema de Salud de Paraguay. Rev. Salud Pública Parag. 2011;1(1):13-25. Recuperado en Junio de 2016 http://www.mspbs.gov.py/aps/wpcontent/ uploads/2013 /11/Sistema-deSalud-del-Paraguay_2011__ Revista-deSalud-Publica-del-INS.pdf, agosto 2015

7. OPS, Organización Panamericana de la Salud. Perfil de los Sistemas de Salud de Paraguay: Monitoreo y análisis de los procesos de cambio y reforma. Washington D.C. 2008. Recuperado en Junio 2016. Disponible en: http://www.mspbs.gov.py/planificacion/wpcontent/uploads/2012/07/Perfil_Sistema_Sal ud-Paraguay_2008.pdf

8. Bossert T. Analyzing the decentralization of health systems in developing countries: decision space, innovation and performance. Social science \& medicine. 1998;47(10):1513-27.

http://EconPapers.repec.org/RePEc:eee:soc med:v:47:y:1998:i:10:p:1513-1527

9. Bossert T. Methodological Guidelines for Applied Research on Decentralization of Health Systems in Latin America. Harvard School of Public Health, Data for Decision Making (DDM); 2000.
10. Giménez E. Hacia un sistema de Salud con Garantías. Notas para nuevas políticas en salud en Paraguay. Instituto Desarrollo / Higea Salud Paraguay; 2013.

11. Nickson A. El gobierno local en Paraguay: Un análisis comparativo a través de diez elementos. ID- Ajumpa, Asunción 2016.

12. Murray CJ, Frenk J. Sistemas de Salud: Un marco para evaluar el desempeño de los Sistemas de Salud. OMS. 2000;3:149-62. http:// whqlibdoc.who.int/boletin/ 2000/ RA_2000_3_149-162_spa.pdf

13. Organización Mundial de la Salud. La atención primaria de salud más necesaria que nunca. Informe sobre la salud en el mundo 2008. Informe de un Grupo Científico de la OMS; Ginebra: OMS; 2008. Serie de informes técnicos: clasificación NLM: W 84.6 ISBN 9789243363732. Disponible en: http://www.who.int/whr/2008/08_report_es .pdf

14. Giménez $\mathrm{E}$, Rodríguez JC, Peralta N. Espacios de decisión y tendencias en la descentralización de salud en el Paraguay. Investigación para el Desarrollo. Asunción Paraguay. En imprenta (2016). 